\title{
Cooperative Self-Organization to Design Robust and Adaptive Collectives
}

\author{
Gauthier Picard and Marie-Pierre Gleizes \\ IRIT - Université Paul Sabatier \\ 118, route de Narbonne - 31062 Toulouse Cedex FRANCE \\ \{picard,gleizes\}@irit.fr
}

Keywords: Multi-agent systems, Cooperation, Self-organization

\begin{abstract}
This paper highlights the benefits of using cooperation as the engine of adaptation and robustness for multiagent systems. Our work is based on the AMAS (Adaptive Multi-Agent System) approach which considers cooperation as a self-organization mechanism to obtain adequate emergent global behaviors for systems coupled with complex and dynamic environments. A multi-robot resource transportation task illustrates the instantiation of a cooperative agent model equipped with both reactive and anticipative cooperation rules. Various experiments underline the relevance of this approach in difficult static or dynamic environments.
\end{abstract}

\section{Introduction}

To provide a self-organizing collective behavior, the parts composing a system need a local criterion to re-organize so as to provide a more adapted function (Heylighen, 1999). Designers must specify this criterion and equip parts to process and handle this criterion. To the first point, the AMAS approach answers by using an artificial socially inspired notion, close to symbiosis (Maturana and Varela, 1994): cooperation (Capera et al., 2003). The second point is fulfilled by using an autonomic agent approach, which provides decision-making capabilities to parts. Cooperative agents aim at avoiding Non Cooperative Situations $(N C S)$ as a generic proscriptive re-organizational rule. The advantage of the AMAS approach is to ensure, by providing adequate cooperative local behaviors, that the collective will provide the adequate and adapted global function. To design such agents, a cooperative agent model is available in the ADELFE method (Bernon et al., 2003). To highlight the relevance of using the cooperative agent model, we propose to develop a multi-robot resource transportation task, in which several similar robots have to transport boxes from a room to another one, by passing through narrow corridors, which produce spatial interferences.

This paper rather focuses on the ability of a system to react to environmental changes and on the observation of emergent phenomena, rather than on the nominal efficiency of the transport task. Section 2 presents the agents of the system -the robots- by developing their different modules and nominal behaviors. Section 3 explains the different cooperation rules robots have to respect to achieve their goals in a more efficient way. An experiment platform has been developed to test the instantiation of the cooperative agent model. Several results are expounded and discussed in section 4 before concluding.

\section{Cooperative Robot Agents}

By using the ADELFE method, robots are described with several modules which represent a partition of their physical, social and cognitive capabilities (Bernon et al., 2003).

The Perceptions Module represents inputs for agents: position of the claim zone, position of the laying zone, a limited perception cone in which objects (robot, box or wall) are differentiable, proximity sensors (forward, backward, left and right), a compass and the absolute spatial position. The environment is discretized as a grid whose cells represent atomic parts on which an object can be situated.

The Actions Module represents outputs from the agents on their environment. Actions for the transporter robots are: rest, pick, drop, forward, backward, left and right. Robots cannot drop boxes anywhere in the environment but only in the laying zone. They cannot directly communicate or drop land marks on 
the environment.

The Skills Module contains knowledge about the task the agent must perform. Skills enable robots to achieve their transportation goals. Therefore, a robot is able to calculate which goal it must achieve in terms of its current state: if it carries a box then it must reach laying zone else it must reach claim zone. As a function of its current goal, the Skills Module provides an action to process to achieve it. Moreover, robots have intrinsic physical characteristics such as their speed, the number of transportable boxes or the preference to move forward rather than backward - as ants have. Such preferences are called reflex values (see 2.2).

The Representations Module contains knowledge about the environment (physical or social). The representations a robot has on its environment are very limited. From its perceptions, it cannot distinguish a robot from another one, but can know if it is carrying a box or not. It also can memorize its past absolute position, direction, goal and action.

The Aptitudes Module enables an agent to choose an action in terms of its perceptions, skills and representations. In terms of the current goal, the Skills Module provides preferences on every action the robot may do. The Aptitudes Module chooses among these actions what will be the next action to reach the goal. Many decision functions can be considered; e.g. an arbitrary policy (the action having the highest preference is chosen) or a Monte Carlo methodbased policy, which is chosen for our example, since it has already been successfully applied to foraging tasks (Topin et al., 1999). Therefore, the Aptitudes Modules can be summed up in a Monte Carlo decision function on the preference vector (the list of action preferences for an agent) provided by the Skills Module. In the same manner, the Cooperation Module provides preference vectors in order to solve the NCS which are described in section 3.

\subsection{Internal Functioning}

During the perception phase of the agents' life cycle, the Perceptions Module updates the values of the sensors, which directly implies changes in the Skills and Representations Modules. Once the knowledge is updated, the decision phase results in an action choice. During this phase, the Aptitudes Module computes from knowledge and proposes action(s) or not. At each time $t$, a robot chooses between different actions that are proposed by the two decision modules (Skills and Cooperation). At time $t$, each action $a c t_{j}$ of the robot $r_{i}$ is evaluated. For each action, this value is calculated in terms of perceptions, representations and reflexes in the case of a nominal behavior:

$$
\begin{aligned}
V_{r_{i}}^{n o m i}\left(a c t_{j}, t\right)= & w p_{r_{i}}\left(a c t_{j}, t\right)+w m_{r_{i}}\left(a c t_{j}, t\right)+ \\
& w r_{r_{i}}\left(a c t_{j}\right)
\end{aligned}
$$

with:

- $V_{r_{i}}^{\text {nomi }}\left(a c t_{j}, t\right)$ represents the value for the action $a c t_{j}$ at time $t$ for the robot $r_{i}$,

- $w p_{r_{i}}\left(a c t_{j}, t\right)$ represents the calculated value in terms of perceptions,

- $w m_{r_{i}}\left(a c t_{j}, t\right)$ represents the calculated value in terms of memory,

- $w r_{r_{i}}\left(a c t_{j}, t\right)$ represents the calculated value in terms of reflexes.

All the $V_{r_{i}}^{\text {nomi }}\left(a c t_{j}, t\right)$ are grouped in a vector, called action preference vector. In the same manner, the Cooperation Module detects if the agent is faced up to a NCS or not. In the former case, the Cooperation Module proposes an action that subsumes the actions proposed by the Aptitudes Module. In the latter case, the action proposed by the Aptitudes Module is chosen and, then, the agent acts by activating effectors and/or changing its knowledge. As for aptitudes, an action preference vector, $V_{r_{i}}^{\text {coop }}\left(a c t_{j}, t\right)$, is generated by the Cooperation Module, by regrouping evaluation of the actions. Once these values have been calculated by the two modules for each action of a robot, the vector on which the Monte Carlo drawing will process is a combination of the two vectors in which the cooperation vector subsumes the nominal vector:

$$
V_{r_{i}}(t)=V_{r_{i}}^{\text {nomi }}(t) \prec V_{r_{i}}^{\text {coop }}(t)
$$

\subsection{Nominal Behavior}

The nominal behavior is described with rules that modify the values in the $V^{n o m i}$ preference vector. This vector is obtained by adding values from perceptions $\left(w p_{r_{i}}\left(a c t_{j}, t\right)\right)$ and values from reflexes $\left(w r_{r_{i}}\left(a c t_{j}, t\right)\right)$. Memory is not necessary to implement a this behavior. Table 1 shows values to increase in the $w p_{r_{i}}\left(a c t_{j}, t\right)$ to achieve the two disjoint goals: reach claim zone ( $\neg$ car) and reach laying zone (car).

Reflex values are static and also depend on perceptions and more precisely on the direction of the robot. As for ants, robots may prefer moving forward then backward (Topin et al., 1999). For example, values for $w r_{r_{i}}\left(a c t_{j}, t\right)$ can be: $w r_{r_{i}}($ forward,$t)=$ $50 ; w r_{r_{i}}($ left,$t)=10 ; w r_{r_{i}}($ right,$t)=10$; $w r_{r_{i}}($ backward, $t)=0$. Thus, even if a goal leads a robot to a wall, the robot can move by side, as ants do to forage and to avoid dead ends. But, this mechanism is not sufficient to avoid deadlocks in long narrow corridors in which robots cannot cross. The goal is more influential than reflexes. As a consequence, defining cooperation rules is necessary to enable all robots to achieve their tasks without deadlock.

\subsection{Cooperative Attitude of Agents}

The AMAS theory identifies several types of NCS (Capera et al., 2003), resulting from the analysis of 


\begin{tabular}{|l|l|}
\hline Perceptions & Effects \\
\hline \hline$\neg \operatorname{car} \wedge c B o x$ & $\nearrow w p_{r_{i}}($ pick,$t)$ \\
\hline$\neg \operatorname{car} \wedge \neg c B o x \wedge s B o x$ & $\nearrow w p_{r_{i}}($ forward,$t)$ \\
\hline$\neg \operatorname{car} \wedge \neg c B o x \wedge \neg s B o x \wedge \neg i n C Z$ & $\nearrow w p_{r_{i}}(\langle$ CZdir $\rangle, t)$ \\
\hline$\neg \operatorname{car} \wedge \neg c B o x \wedge \neg s B o x \wedge i n C Z$ & $\nearrow w p_{r_{i}}($ backward,$t)$ \\
& $\nearrow w p_{r_{i}}($ forward, $)$ \\
& $\nearrow w p_{r_{i}}($ left,$t)$ \\
& $\nearrow w p_{r_{i}}($ right,$t)$ \\
\hline \hline $\operatorname{car} \wedge c L Z$ & $\nearrow w p_{r_{i}}($ drop,$t)$ \\
$\operatorname{car} \wedge \neg c L Z$ & $\nearrow w p_{r_{i}}(\langle$ LZdir $\rangle, t)$ \\
\hline with: &
\end{tabular}

- $c a r: r_{i}$ is carrying a box,

- $c B o x: r_{i}$ is close to a box,

- $s$ Box: $r_{i}$ is seeing a box,

- inCZ: $r_{i}$ is in the claim zone,

- $c L Z: r_{i}$ is close to the laying zone,

- $c L Z: r_{i}$ is close to the laying zone,

- $\langle C Z$ dir $\rangle:$ the move to perform to go to claim zone,

- $\langle L Z$ dir $\rangle:$ the move to perform to go to laying zone,

- $\nearrow$ : increasing.

Table 1: Specification of the nominal behavior

the cooperation definition which is close to the symbiosis notion. An agent is cooperative if: all perceived signals are understood without ambiguity $\left(c_{p e r}\right)$ and the received information is useful for the agent's reasoning $\left(c_{d e c}\right)$ and reasoning leads to useful actions toward other agents $\left(c_{a c t}\right)$. Therefore, a NCS occurs when $\neg c_{\text {per }} \vee \neg c_{\text {dec }} \vee \neg c_{a c t}$. We identify seven NCS subtypes that express these conditions:

- incomprehension $\left(\neg c_{\text {per }}\right.$ ): the agent cannot extract the semantic contents of a received stimulus,

- ambiguity $\left(\neg c_{p e r}\right)$ : the agent extracts several interpretations from a same stimulus,

- incompetence $\left(\neg c_{d e c}\right)$ : the agent cannot benefit from the current knowledge state during the decision,

- unproductiveness $\left(\neg c_{d e c}\right.$ ): the agent cannot propose an action to do during the decision,

- concurrency $\left(\neg c_{a c t}\right)$ : the agent perceives another agent which is acting to reach the same world state,

- conflict $\left(\neg c_{a c t}\right)$ : the agent believes the transformation it is going to operate on the world is incompatible with the activity of another agent,

- uselessness $\left(\neg c_{a c t}\right)$ : the agent believes its action cannot change the world state or it believes results for its action are not interesting for the other agents.

Cooperative agents must avoid or repair these NCS, if they occur. Designing such agents focuses on NCS specification -a kind of exception-oriented programming in which designers focus on exceptions.

\section{Cooperative Self-Organization}

Beyond two robots, acting to transport boxes in a same environment, the nominal behavior is no more adequate. Indeed, a robot is equipped with skills to achieve its tasks, but not to work with other robots. In a constrained environment, spatial interference zones appear. If two robots, a first one carrying a box and moving to the laying zone and a second one moving to the claim zone to pick up a box, meet in a corridor, the circulation is blocked.

\subsection{Reactive Cooperation}

Two main NCS can be reactively solved, without requiring memory:

A robot is blocked. A robot $r_{1}$ cannot move forward because it is in front of a wall or another robot $r_{2}$ moving in the opposite direction ${ }^{1}$. In this case, if it is possible, $r_{1}$ must move to its sides (left or right). This corresponds to increasing values of the cooperative action vector related to side movements: $V_{r_{1}}^{\text {coop }}(t, r i g h t)$ and $V_{r_{1}}^{\text {coop }}(t$, left $)$. If $r_{1}$ cannot laterally move, two other solutions are openned. If $r_{2}$ has an antagonist goal, the robot which is the most distant from its goal will move backward (increasing $V_{r_{i}}^{\text {coop }}(t$, backward $)$ ) to free the way for the robot which is the closest to its goal (increasing $V_{r_{i}}^{\text {coop }}(t$, forward $)$ even if it may wait). Robots can evaluate which is the most distant since they know their goals and the associated zones. If $r_{2}$ has the same goal than $r_{1}$, except if $r_{1}$ is followed by an antagonist robot or if $r_{1}$ moves away from its goal (visibly it moves to a risky ${ }^{2}$ region), $r_{1}$ moves backward; else $r_{1}$ moves forward and $r_{2}$ moves backward.

A robot is returning. A robot $r_{1}$ is returning ${ }^{3}$ as a consequence of a traffic blockage. If it is possible, $r_{1}$ moves to its sides (and is not returning anymore). Else, $r_{1}$ moves forward until it cannot continue or if it encounters another robot $r_{2}$ which is returning and is closer to its goal than $r_{1}$. Table 2 sums up the cooperative behavior in this situation. If there is a queue of robots, the first returning robot is seen by the second one that will return too. Therefore, the third one will return too and so on until there are no more obstacles.

These two rules correspond to resource conflicts (on corridors) or uselessness (when robots move backward and away from their goal) situations. These rules, which are simple to express, ensure that robots cannot block each other in corridors, and robots do not need to communicate. But, this cooperative attitude only solves problem instantly, creating returning movements and then implying time loss.

\footnotetext{
${ }^{1}$ If $r_{2}$ moves in another direction than the opposite direction of $r_{1}$, it is not considered as blocking because it will not block the traffic anymore on the next step.

${ }^{2}$ It is risky in the sense it may lead to the occurrence of a lot of non cooperative situations such as conflicts.

${ }^{3} \mathrm{~A}$ robot is considered as returning while it has no choice of side movements.
} 


\begin{tabular}{|c|c|}
\hline Condition & Action \\
\hline ret $\wedge f R$ & $\nearrow V_{r_{i}}^{\text {coop }}(t, r i g h t)$ \\
\hline ret $\wedge f L$ & $\nearrow V_{r_{i}}^{c o o p}(t, l e f t)$ \\
\hline $\begin{array}{l}\text { ret } \wedge \neg(f L \vee f R) \wedge a n t \wedge \text { toGoal } \wedge \\
\text { cGoal }\end{array}$ & $\nearrow V_{r_{i}}^{c o o p}(t$, backward $)$ \\
\hline $\begin{array}{l}\text { ret } \wedge \neg(f L \vee f R) \wedge a n t \wedge \text { toGoal } \wedge \\
\neg c G o a l\end{array}$ & $\nearrow V_{r_{i}}^{\text {coop }}(t$, forward $)$ \\
\hline ret $\wedge \neg(f L \vee f R) \wedge$ ant $\wedge \neg$ toGoal & $\nearrow V_{r_{i}}^{\text {coop }}(t$, backward $)$ \\
\hline ret $\wedge \neg(f L \vee f R) \wedge \neg$ ant & $\nearrow V_{r_{i}}^{\text {coop }}(t$, forward $)$ \\
\hline
\end{tabular}

- ret: $r_{i}$ is returning,

- $f R$ : right cell is free

- $f L$ : left cell is free,

- ant: in front of an antinomic robot,

- toGoal: $r_{i}$ is moving to goal,

- cGoal: $r_{i}$ is closer to its goal than its opposite one,

- $\nearrow$ : increasing.

Table 2: Specification of "a-robot-is-returning" NCS.

\subsection{Anticipative Cooperation}

It is possible to specify cooperation rules to anticipate blockage situations in order to make the collective more efficient : these rules are optimization cooperation rules. Previous rules enable robots to extract from blockage. A robot is in such a situation because it was crossing a zone frequented by antinomic robots. So as to prevent this situation and to avoid repeating their past non cooperation failures, robots must memorize locations of risky areas (from which antinomic robots come) and avoid them. Thus, an anticipation rule can be specified:

A robot sees an antinomic robot. If a robot $r_{1}$ perceives a robot $r_{2}$ having an antinomic goal and if $r_{1}$ can move to its sides it does else it moves forward.

Nevertheless, this reactive anticipation presents a major problem: once a robot has avoided the risky zone, no mechanism ensures that it will not go in it again, led by its goal. Robots can be equipped with a limited memory of the risky zones (in the Representations Module). Each time $t$ a robot $r_{i}$ experiments an anticipation situation facing a robot $r_{j}$, it memorizes a tuple (or virtual marker) $\left\langle\operatorname{pos} X\left(r_{j}, t\right), \operatorname{pos} Y\left(r_{j}, t\right), \operatorname{goal}\left(r_{i}, t\right), w\right\rangle$ in which $\operatorname{pos} X\left(r_{i}, t\right)$ and $\operatorname{pos} Y\left(r_{i}, t\right)$ represent the coordinates of $r_{j}$ at the moment $t$. goal $\left(r_{i}, t\right)$ represents the goal $r_{i}$ was achieving at time $t$. $w$ represents a repulsion value. The higher this value is, the more the robot will try to avoid the zone described by the marker when it is achieving another goal than $\operatorname{goal}\left(r_{i}, t\right)$. Therefore, the robot inspects all its personal markerswhose distance is less than its perception limit (to fulfill the locality principle). A marker with a weight $w$ and situated in the direction $\operatorname{dir}$ at a distance $d$ induces that $V_{r_{i}}^{\text {coop }}\left(t, \operatorname{dir}_{\text {opp }}\right)$ will be increased of $w\left(\right.$ dir $_{\text {opp }}$ is the opposite direction to dir $)$.

As the memory is limited, tuples that are added

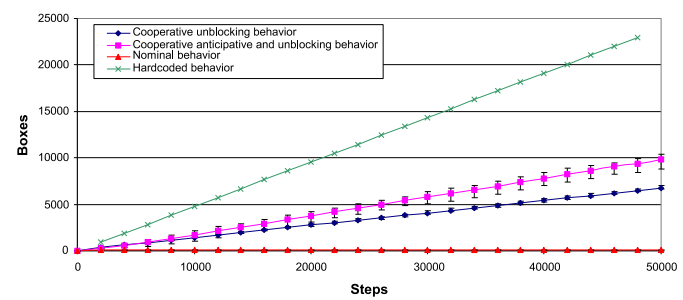

Figure 1: Average number of returned boxes in terms of simulation time and standard deviation for 15 simulations for different behaviors: individualist, cooperative unblocking, cooperative anticipative and hardcoded behavior.

must disappear at runtime. The weight $w$ decreases of a given value $\delta_{w}$ (called forgetting factor) at each step. Once $w=0$, the tuple is removed from the memory. This method corresponds to the use of virtual and personal pheromones, and, as ants do, robots reinforce their markers: a robot moving to a position corresponding to one of its marker with another goal, re-initializes the marker. But contrary to the ant approach, no stigmergy or physical medium are needed.

\section{Experiments and Discussion}

The expounded model has been implemented and simulated in the environments shown in figures 3, 4 and 5, with the different entities : boxes (non bordered circles), robots without boxes (light grey bordered circles) and robots carrying boxes (dark grey bordered circles). This environment is made up of two rooms $(25 \times 30$ cells $)$ separated by two long and narrow corridors $(30 \times 1$ cells $) .300$ robots are randomly placed in the claim room. These robots can perceive at a distance of 5 cells, and can make a move of one cell at each step. If they can anticipate conflicts, their memory can contain 1500 tuples with an initial repulsion value $w=400$ and a decrease value $\delta_{w}=1$.

Reaction vs. Anticipation. Figure 1 shows a comparison between the average numbers of transported boxes for 15 simulations (300 robots, 2 corridors, 5ranged perception), corresponding to the nominal behavior (individualist), a hardcoded one and two cooperative ones: the cooperative unblocking behavior (see section 3.1) and the cooperative anticipative behavior (see section 3.2). By adding blockage anticipation, the collective becomes more efficient (at least $30 \%$ more boxes are transported). According to the AMAS paradigm, we can experimentally observe that the local resorption of NCS leads to the collective functional adequacy. Nevertheless, equipping agents with cooperative behavior is not as efficient as hardcoding by giving non adaptive passage points: claim 

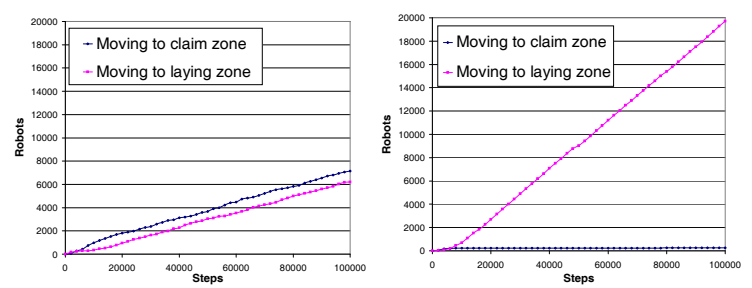

Figure 2: Incoming robots for the top corridor for two different behaviors: unblocking behavior (left) and anticipative unblocking behavior (right).

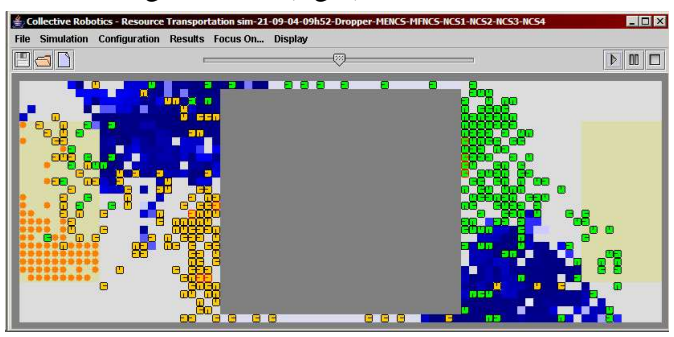

Figure 3: Positioning of all the virtual markers (dark squares) for all the robots and the two goals.

zone, then corridor 1, then laying zone, then corridor 2 , as shown in figure 1. Finally, the global adaptive behavior is not sensible to initial state as shown by the standard deviation, which is negligible.

Emergence of Corridor Dedication. Figure 2 shows the corridor-usage for the two cooperative behaviors, i.e. the number of incoming robots for a corridor and for the two cooperative behaviors: unblocking behavior (left) and anticipative unblocking behavior (right). In the case of anticipative behavior, we can observe the emergence of a sense of traffic. Robots collectively dedicate corridors to particular goals. In fact, markers of all the agents are positioned only at one corridor entry for one direction as shown in figure 3. This view is only for monitoring purpose: robots do not perceives all the markers, only their owns. We can assign the emergent property to this phenomenon because robots do not handle any notion of corridor - unlike some previous works (Picard and Gleizes, 2002). Thus, just with local data, robots established a coherent traffic behavior that leads to an optimization of the number of transported boxes.

Robustness in Difficult Environments. Some simulations have been done in the environment with dead ends of figure 4 . Robots are able to manage such difficulties by using directions at a higher abstraction level. For example, the forward direction is not simply interpreted as going straight forward. If the robot has only one free direction, left for example, going forward will become going left. As a consequence, a

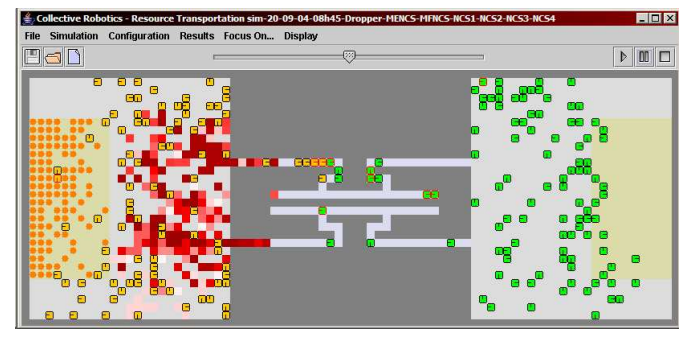

Figure 4: Positioning of all the virtual markers (dark squares) for the goal reach laying zone in a difficult environment (with deadends).

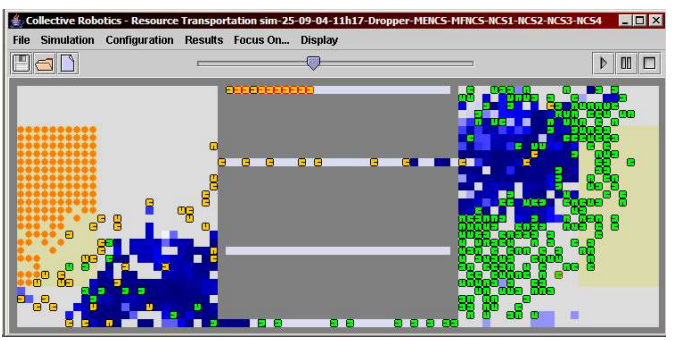

Figure 5: Positioning of the virtual markers (dark squares) in a dynamic environment with two closed corridors.

dead end problem is easily avoided. But, robots do not distinguish corridors, since they are too close and markers are not precise enough. In our example, corridors are separated by only 9 cells, which is less then two times the perception distance of robots. Therefore, marked areas intersect and robots cannot precisely detect which corridor to avoid. Thus, there is no corridor dedication, as shown in figures 4. However, the collective behavior is robust enough so that no deadlock appears, even if the collective becomes less efficient, because the learning process is disabled. Solving this problem implies either modifying parameters such as distance of perception or repulsing force of markers in function of the environment (which is too environment-dependent), or adding learning capabilities on these parameters by adding new NCS rules.

Adaptation to Dynamics. Simulations have been done with an environment with four corridors. At each 10,000 step period, two corridors are randomly chosen and closed to add non determinism and dynamics. Here again, the collective is less efficient, but there is no deadlock. Of course, if corridor closing is too frequent, robots cannot adapt and dedicate directions to corridors. In fact, 300 robots need about 2000 steps to adapt. Figure 5 shows that only entries of opened corridors are marked and some robots can be captured in closed corridors, and are then useless. During each closing period, the two opened corridors are exploited and a direction is affected. Moreover, the effect of the environmental dynamic become neg- 


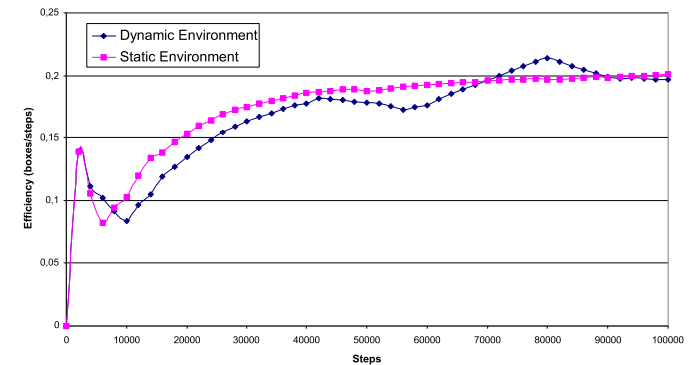

Figure 6: Compared efficiency (boxes/time) between a simulation in a static environment with two corridors and another one in an dynamic environment with 4 corridors which 2 corridors are randomly closed every 10,000 steps.

ligible with a great number of steps. Figure 6 shows the efficiency for two different environment (static or dynamic). The efficiency of the system in the static one is more stable but equivalent to the efficiency of the system which evolves in the dynamic one.

Discussion. The AMAS cooperative agent approach is relevant for several reasons. Firstly, unlike ant algorithms (Bonabeau et al., 1997), robots do not physically mark their environment with pheromones, but memorize personal markers. Secondly, contrary to competition-based (Vaughan et al., 2000) or altruistic (Lucidarme et al., 2002) approaches, robots do not need direct communication to inform close robots or exchange requests and intentions. Thirdly, cooperative behavior encoding is insensitive to the number of robots, to the topography and to the dimensions of the environment. Fourthly, no global feedback is needed to lead the system to functional adequacy, which prevents the system to reach local extrema. Finally, obtained collectives are robust and adaptive, even if perception capabilities are very limited. Nevertheless, ADELFE does not provide any guidance to instantiate these values. For instance, the initial weights for markers and the forgetting factor have been adjusted to the time robots spend to cross the entire environment. This might be completely different in a more complex environment with more or less corridors which can dynamically open or close. Some simulations have been done with such environments, and the affected values seem correct unless corridors are too close to each other or frequency of closure is too fast. These values also may be learnt at runtime, which is one of our perspectives.

\section{Conclusion}

In this paper, we have shown the relevance of cooperation as a local criterion for collectives to self- organize to be more adapted to a specific task. Considering the ignorance of the global task and the environment, the self-organizing collective reaches an emergent coherent behavior, which is then more robust to environmental risks. Our simulation application tackles a simple problem in a simple static environment in which the collective achieves its global task. Simulations in difficult and dynamic environments confirm the relevance of cooperative selforganizing collectives. Finally, this application has been developed to confront the ADELFE cooperative agent model to a task that requires adaptation and robustness. However, this application is very specific, in the sense it only concerns quasi-reactive non communicative robots. In the case of agents able of communicative acts, other kinds of NCS are possible.

\section{REFERENCES}

Bernon, C., Camps, V., Gleizes, M.-P., and Picard, G. (2003). Designing Agents' Behaviors and Interactions within the Framework of ADELFE Methodology. In $4^{\text {th }}$ Engineering Societies in the Agents World (ESAW'03), volume LNCS 3071, pages 311327. Springer-Verlag.

Bonabeau, E., Theraulaz, G., Deneubourg, J.-L., Aron, S., and Camazine, S. (1997). Self-organization in social insects. Trends in Ecology and Evolution, 12:188193.

Capera, D., Georgé, J., Gleizes, M.-P., and Glize, P. (2003). The AMAS theory for complex problem solving based on self-organizing cooperative agents. In $1^{\text {st }}$ Int. TAPOCS Workshop at $12^{\text {th }}$ IEEE WETICE, pages 383-388. IEEE.

Heylighen, F. (1999). The Science of Self-organization and Adaptivity. EOLSS Publishers.

Lucidarme, P., Simonin, O., and Liégeois, A. (2002). Implementation and Evaluation of a Satisfaction/Altruism Based Architecture for Multi-Robot Systems. In IEEE Int. Conf. on Robotics and Automation (ICRA'02), pages 1007-1012.

Maturana, H. and Varela, F. (1994). The Tree of Knowledge. Addison-Wesley.

Picard, G. and Gleizes, M.-P. (2002). An Agent Architecture to Design Self-Organizing Collectives: Principles and Application. In AISB'02 Symposium on Adaptive Multi-Agent Systems (AAMASII), volume LNAI 2636, pages 141-158. Springer-Verlag.

Topin, X., Fourcassié, V., Gleizes, M.-P., Theraulaz, G., Régis, C., and Glize, P. (1999). Theories and experiments on emergent behaviour : From natural to artificial systems and back. In European Conf. on Cognitive Science, Siena, Italy.

Vaughan, R., Støy, K., Sukhatme, G., and Matarić, M. (2000). Go ahead make my day: Robot conflict resolution by aggressive competition. In $\sigma^{\text {th }}$ Int. Conf. on Simulation of Adaptive Behaviour. 